\title{
Precision Monte Carlo Test of the Hartree-Fock Approximation for a trapped Bose Gas
}

\author{
Markus Holzmann and Werner Krauth * \\ CNRS-Laboratoire de Physique Statistique de l'Ecole Normale Supérieure, 24, rue Lhomond, 75231 Paris Cedex 05, France
}

\author{
Martin Naraschewski * \\ Jefferson Laboratory, Department of Physics, Harvard University, Cambridge MA 02138, USA \\ ITAMP, Harvard-Smithsonian Center for Astrophysics, 60 Garden Street, Cambridge, MA 02138, USA
}

\begin{abstract}
We compare the semiclassical Hartree-Fock approximation for a trapped Bose gas to a direct Path Integral Quantum Monte Carlo simulation. The chosen parameters correspond to current ${ }^{87} \mathrm{Rb}$ experiments. We observe corrections to the mean-field density profile. The Path Integral calculation reveals an increase of the number of condensed particles, which is of the same order as a previously computed result for a homogeneous system. We discuss the experimental observability of the effect and propose a method to analyze data of in-situ experiments.
\end{abstract}

PACS numbers: 03.75.Fi, 02.70.Lq, 05.30.Jp

The experimental realization of Bose-Einstein condensation (BEC) in dilute atomic vapors [1] has generated extraordinary experimental and theoretical interest. From the viewpoint of quantum many-body physics, the trapped atomic vapors are peculiar. Well above the critical point, the gases are extremely dilute, and their description as noninteracting bosons is very accurate. As the condensation sets in, the trapped atoms are strongly compressed in real space. Then, interactions become much more important and even the simplest thermodynamic quantities (spatial distribution, condensate fraction, etc.) have to be obtained by the appropriate quantum many-body technique. At zero temperature the Bogoliubov approach of weakly interacting Bose gases is well established [4. There, the macroscopic condensate wave function is given by the Gross-Pitaevskii equation [5]. The effects of noncondensed particles at finite temperatures can be included via the Hartree-Fock-Bogoliubov equations, which have been solved in the Popov approximation [6] and in various simplified forms [7].

The equilibrium properties of Bose gases can also be directly computed by Path Integral Quantum Monte Carlo (QMC) simulation without any essential approximation [8]. The QMC approach was used to first show [9] that the trapped Bose gas with repulsive interactions has a lower critical temperature $T_{c}$ and a smaller number $N_{0}$ of condensed particles below $T_{c}$ than the free Bose gas, one of the predictions of mean-field theories [10,11].

Very importantly, the QMC calculation is free of systematic errors: it gives a somewhat noisy, but otherwise exact numerical solution of BEC. For simulations corresponding to dilute atomic vapors, the QMC calculation can be performed directly for the large particle numbers $\left(\sim 10^{4}-10^{5}\right)$ and the temperatures of experimental in- terest. In this situation, there is little need to perform finite-size scaling. QMC provides us thus with a unique opportunity to check the above-mentioned many-body techniques directly in an experimental setting, a comparison which we provide here.

It is the main point of this paper to study the corrections of a full many-particle treatment to a mean-field theory. We compare the QMC calculations to the semiclassical Hartree-Fock (HF) approximation, which determines the local density in accordance with the local trap potential and the mean interaction energy between the particles. The HF approximation provides an ideal reference system, since it corresponds locally to a homogeneous ideal Bose gas. The corrections to HF should be therefore comparable to previous calculations in a homogeneous system 12 14].

For a system closely corresponding to many experiments with ${ }^{87} \mathrm{Rb}$ we find deviations from the HF density which are concentrated in the overlap region between the excited atoms and the condensate. The number of condensed particles $N_{0}$ is increased around the critical temperature by about $5 \%$. We also discuss a scheme for a detailed analysis of experimental in-situ measurements.

The QMC calculation is beset with statistical fluctuations. In fact, the noise of independent Monte Carlo configurations reproduces the sample-to-sample variations of repeated experimental measurements at the same temperature $T$ and particle number $N$ (or chemical potential). This allows us to discuss the experimental observability of these corrections to mean-field theory.

The Hamiltonian of $N$ interacting particles in an isotropic harmonic trap with frequency $\omega$ is given by

*holzmann@physique.ens.fr; krauth@physique.ens.fr; martin@mistral.harvard.edu 


$$
H=\sum_{i=1}^{N}\left[\frac{p_{i}^{2}}{2 m}+\frac{1}{2} m \omega^{2} r_{i}^{2}\right]+\frac{1}{2} \sum_{i, j=1}^{N} V\left(r_{i j}\right),
$$

where $V$ is the interatomic (pseudo-)potential between two particles. A hard-core interaction with radius $a$ is customary.

The partition function $Z$ of the system with inverse temperature $\beta=\left(k_{B} T\right)^{-1}$ is given by the trace of the symmetrized density matrix $\rho=e^{-\beta H}$ over all states. $Z$ satisfies the usual convolution equation:

$$
\begin{array}{r}
Z=\frac{1}{N !} \sum_{P} \int d R \rho\left(R, R^{P}, \beta\right)=\frac{1}{N !} \sum_{P} \times \\
\times \int d R \int d R_{2} \cdots \int d R_{M} \rho\left(R, R_{2}, \tau\right) \cdots \rho\left(R_{M}, R^{P}, \tau\right) .
\end{array}
$$

Here $\tau=\beta / M, R$ is the $3 \mathrm{~N}$-dimensional vector of the particle coordinates $R=\left(r_{1}, r_{2}, \ldots, r_{N}\right)$, and $R^{P}$ denotes the vector with permuted labels: $R^{P}=$ $\left(r_{P(1)}, r_{P(2)}, \ldots, r_{P(N)}\right)$ [15]. As explained elsewhere [8] the QMC calculation relies on virtually exact formulas for the density matrices $\rho\left(R, R^{\prime}, \tau\right)$ at the higher temperature $1 / \tau$ and performs the integral over $R, R_{2}, \ldots, R_{M}$ as well as the sum over all permutations $P$ in Eq. (2) by Monte Carlo sampling. Special data-handling techniques allow to cope with very large atom numbers $N$.

As in [9] we consider a model system of 10,000 particles, correponding to a critical temperature of $k_{B} T_{c}^{0} \simeq$ $20.25 \hbar \omega$ and a hard-core potential with radius $a=$ $0.0043 a_{0}\left(a_{0}=(\hbar / m \omega)^{1 / 2}\right)$. These values are typical for most ${ }^{87} \mathrm{Rb}$ experiments which are now in operation. We generally perform computations at different values of $\tau$, and extrapolate to $\tau \rightarrow 0$, in which limit the QMC formulas for the density matrices in Eq. (2) become manifestly exact.

In BEC, a single quantum state is occupied by a very large number of bosons. In the presence of interactions this state is a complicated many-body wave function. As far as one particle properties are concerned it can, however, be described by a single particle wave function $\psi_{0}(r)$ determined by the modified Gross-Pitaevskii equation

$$
\left(-\frac{\hbar^{2} \nabla^{2}}{2 m}+\frac{m \omega^{2}}{2} r^{2}+U\left[n_{0}(\mathbf{r})+2 n_{T}(\mathbf{r})\right]-\mu_{0}\right) \psi_{0}(\mathbf{r})=0
$$

where the densities of condensed $n_{0}(\mathbf{r})=N_{0} \times\left|\psi_{0}(\mathbf{r})\right|^{2}$ and thermally excited particles $n_{T}(\mathbf{r})$ account for interactions between these particles. The strength of the interaction is given by $U=4 \pi \hbar^{2} a / m$, where $a$ denotes again the hard sphere radius. The factor of 2 in front of $n_{T}$ accounts for quantum statistical exchange energy. In the semiclassical HF approximation the thermal density is given by [10,11]

$$
n_{T}(\mathbf{r})=\frac{1}{\lambda_{T}^{3}} g_{3 / 2}\left(e^{-\left(m \omega^{2} r^{2} / 2+2 U n(\mathbf{r})-\mu_{T}\right) / k_{B} T}\right) .
$$

Here, the thermal wavelength $\lambda_{T}=\hbar \sqrt{2 \pi / m k_{B} T}$ and the Bose function $g_{3 / 2}(z)=\sum_{j=1}^{\infty} z^{j} / j^{3 / 2}$ have been used. In order to obtain the density distributions of the condensate and of the thermal component, Eqs (3) and (4) have to be solved conjointly at the same chemical potential $\mu=\mu_{0}=\mu_{T}$ with the constraint of the fixed particle number $N$ (different notations for the chemical potential appear for later convenience, cf. below). HF neglects the collective excitations of a more fundamental Bogoliubov theory. However, it was observed that these excitations do not contribute significantly to thermodynamic properties at temperatures well above the chemical potential [16]. On the other hand, collective excitations are fully included in our QMC calculation, as recently confirmed by a comparison of two-particle correlation functions 17].

The precise solution of the HF equation is quite difficult. We have obtained identical results both with an iterative procedure [16] and an interpolation-minimization routine cf. 9]. In a small window around the transition temperature, the HF equations have no solution.

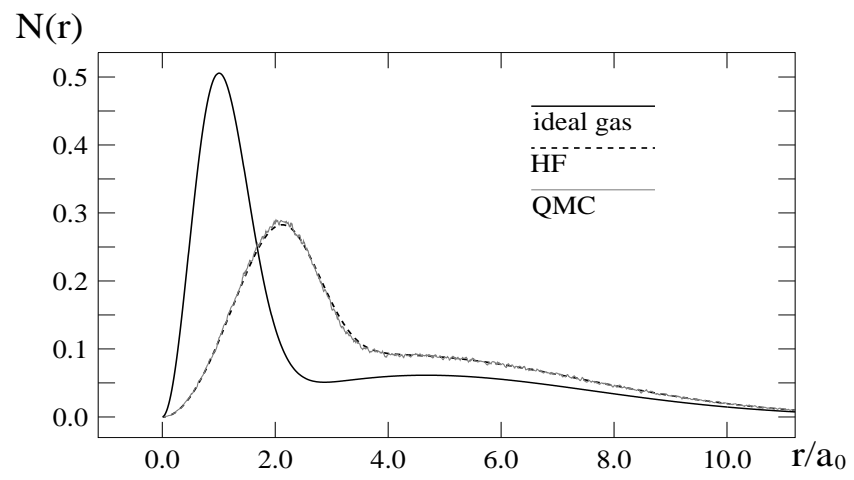

FIG. 1. Number density $N(r)$ from $\mathrm{HF}$ and the exact QMC calculations compared to the non-interacting Bose gas, both for $N=10,000$ particles in an isotropic trap with $\beta=0.07 / \hbar \omega\left(T \simeq 0.7 T_{c}^{0}\right)$.

The raw output of our Monte Carlo simulations consists in histograms of $N(r)$, the number of particles at a distance $r$ from the center of the trap. In Fig. 1, we compare the QMC results for $N(r)$ with the HF solution far below the critical temperature. We notice an excellent agreement between the two approaches. For comparison we also show the result for 10,000 non-interacting bosons at the same parameters. The difference is very important. In Fig. 2, we show corresponding data for a temperature closer to the critical point. Important qualitative information can be obtained directly from Fig. 1 and Fig. 2 without further data analysis [18] i) with respect to the non-interacting solution, the number of condensed particles (both for HF and QMC) is decreased, while their distribution is widened. This can be seen from the first 
peak in $N(r)$, which is shifted to larger values of $r$. Notice that, even far away from the center of the trap, the distribution of the interacting gas is quantitatively different from the ideal gas. We have checked that a temperature determination of these atoms within the ideal gas model fails by about $5 \%$. ii) With respect to the mean-field solution, the number of condensed particles in the exact numerical calculation is increased. This second point nicely checks with recent QMC [12] and renormalization group calculations 13 for the isotropic case. These calculations indicate that the degeneracy parameter is changed by interaction effects beyond mean-field and that for a dilute gas the critical temperature is increased linearly in $a / \lambda_{T}$. It might be suspected that this increase is a mere consequence of quasi-particle excitations. However, it has been shown within the Popov approximation that quasi-particles lead rather to a minute decrease of the critical temperature [16].

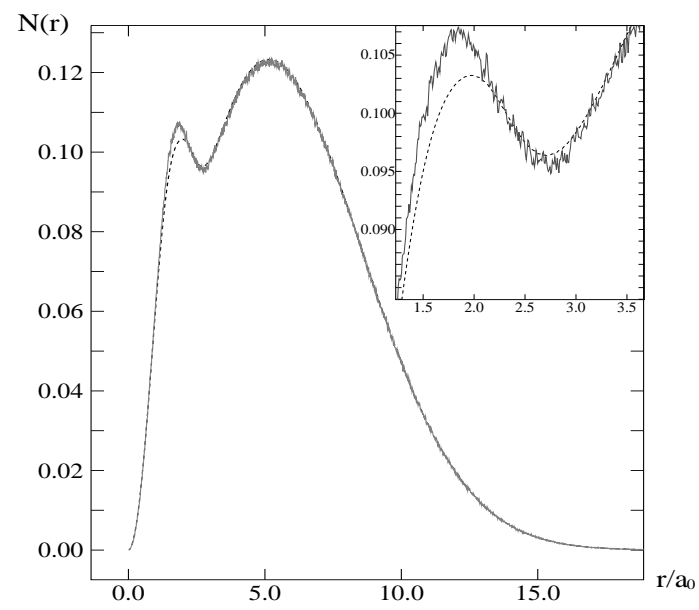

FIG. 2. $\quad N(r)$ from HF and the exact QMC calculations for the same system as in Fig. 1, but at a higher temperature, $\beta=0.056 / \hbar \omega\left(T \simeq 0.88 T_{c}^{0}\right)$. The inset shows the two curves in the overlap region.

To analyze the data we have extracted the number of condensed particles as well as their distribution function from the QMC calculation. In Ref. [9] both were obtained from the permutation-cycle lengths (cf. also 19 ). Here, however, we have preferred to directly "fit" the QMC-generated histograms of $N(r)$ from a very large number of samples to smooth curves $n_{0}(r)$ and $n_{T}(r)$, as has been done to analyze experimental in situ measurements. We have found a nearly perfect fit to the data, on the local density approximation level, by using functional forms for $n_{0}(r)$ and $n_{T}(r)$ as given in Eqs (3) and (4) with unrestricted 'chemical potentials' $\mu_{0}$ and $\mu_{T}$. Eqs (3) and (4) are used, but the fit procedure is not a HF approximation in disguise. This can be seen by a simple counting argument: In the HF calculation, the single parameter $\mu$ allows to satisfy the constraint on the total number of particles. The HF solution, whenever it exists, usually is unique. In the present case, under the same conditions, we have one more parameter, which we fix by the condition of minimizing the meansquare displacement between the data points and the interpolating function. The $\chi^{2}$ obtained was compatible with purely statistical deviations for histograms containing more than $10^{7}$ data points. We note in passing that the density $\rho(r)=N(r) / 4 \pi r^{2}$ and the component densities are obtained with very good precision after performing the fit of the raw-data histogram rather than from a direct rescaling of the data.

This fit could also provide a scheme for the accurate determination of $T$ within experimental in-situ measurements. In current experiments the temperature is mostly determined by a time-of-flight method. Due to collisions during the expansion and the non-instantaneous switchoff of the trap this method limits the temperature determination to $5 \%$, so that a more accurate determination of the critical temperature and the condensate fraction has not yet been possible [20,21].

The use of the fit allows us to compute $N_{0}$ vs. temperature for the set of parameters chosen. The results, extrapolated to $\tau \rightarrow 0$, are plotted in Fig. 3 together with the HF result, which gives a consistently smaller value of $N_{0}$. It should be noticed that the HF equations, as mentioned, have no solution for a range of temperatures close to $T_{c}$. In fact, on approaching this window from below the effective chemical potential $\mu_{\mathrm{eff}}=-m \omega^{2} r^{2} / 2-2 U n(r)+\mu_{0}$

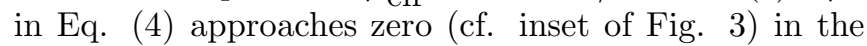
overlap region of the two components. Since the Bose function $g_{3 / 2}(z)$ diverges for $z>1$, this causes a discontinuity of the HF solution. This problem is a finite size effect which is caused by the nonvanishing kinetic energy of the condensate wave function.

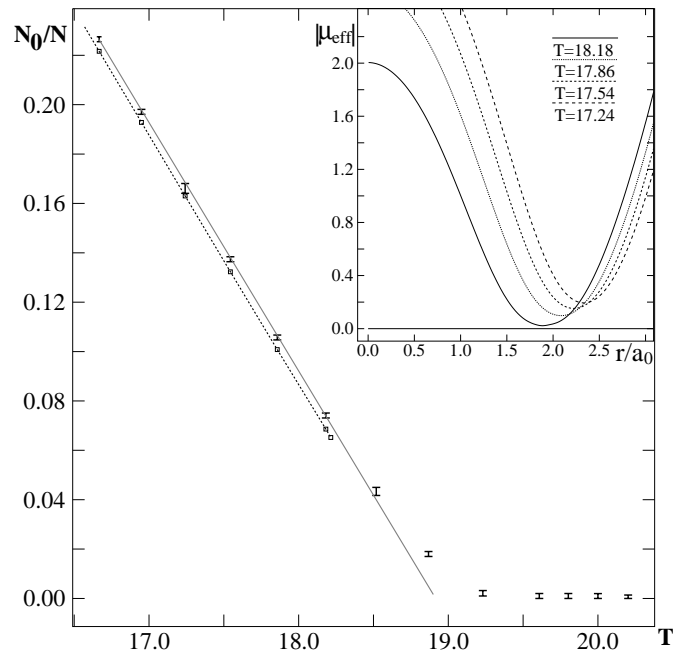

FIG. 3. Extrapolated condensate fraction $N_{0} / N$ from $\mathrm{HF}$ (lower) and from QMC (upper) vs $T$ in units of $\hbar \omega$. The grey line interpolates the QMC data. The inset illustrates the instability of the HF solution as the effective chemical potential $\left|\mu_{\text {eff }}(r)\right|$ approaches zero.

The correction to mean-field theory shown in Fig. 3 
provides definite support for the scenario found in the spatially homogeneous Bose gas: Interactions lead to an increased tendency towards Bose condensation [12, 13. There is however an important difference of context: in a homogeneous system the density at each point is unchanged by interactions and the shift in $T_{c}$ corresponds directly to corrections to HF. As we have seen in the present case the trap is much more complicated since the finite-size effects, the mean-field itself, and finally the corrections to mean-field all influence the density profile and the condensate fraction. However, the finitesize effects and the mean-field itself are already included in HF. Therefore the difference between QMC and HF become directly comparable to the homogeneous system. In Ref. [12], the following behavior is proposed: $\delta T_{c} / T_{c}^{0}=\left(T_{c}-T_{c}^{0}\right) / T_{c}^{0} \simeq 0.34 a \rho^{0.34} \simeq 0.46 a / \lambda_{T_{c}^{0}}$. In our case we would expect $\delta T_{c} \sim 0.065$. This corresponds roughly to the apparently constant offset in $T$ of the two lines in Fig. 3.

It has been suggested [14] that a direct measurement of the density in the center of the trap $\rho(r=0)$ would be useful to determine the deviation of the degeneracy parameter from the mean-field result, $\rho(0) \lambda_{T_{c}}^{3}=2.61$. Even though we are able to extract $\rho(0)$ from QMC or from experimental data very precisely, the onset of condensation is not very sharp and the finite-size effects prevent a precise determination of the critical degeneracy parameter [22]. We rather advocate a direct comparison between $\mathrm{HF}$ (which is available for finite systems) and the data.

It is evident from an experimental point of view that the observation of these corrections necessitates a high resolution for $T$ and $N$. Even for ideal experiments the intrinsic sample-to-sample fluctuations may prevent their observability. Within QMC, we have determined these fluctuations from repeated measurements of the complete distribution of one whole sample. To be able to distinguish clearly the mean particle distribution from the mean-field result, one single configuration of the 10,000 particles is not sufficient. We find that an average of at least 50 independent samples has to be taken in order to detect that there is a difference between $\mathrm{HF}$ and the exact result in the sense of a Kolmogorov-Smirnov test [23]. For a precise evaluation (as in Fig. 3) a much larger data pool is necessary. Experimentally this seems to require a nondestructive measurement scheme.

In conclusion, we are convinced that comparison between theory and experiment on the level treated in this paper is conceivable. An experimental access to the mean-field corrections will be of outstanding theoretical interest [24].

Fruitful discussions with Franck Laloë are acknowleged. This work was partially supported by the EC (TMR network ERBFMRX-CT96-0002), the Deutscher Akademischer Austauschdienst, and the Deutsche Forschungsgemeinschaft. We are grateful to the ITP, Santa Barbara for hospitality.
[1] M. H. Anderson, J. R. Ensher, M. R. Matthews, C. E. Wieman, and E. A. Cornell, Science 269, 198 (1995).

[2] K. B. Davis, M.-O. Mewes, M. R. Andrews, N. J. van Druten, D. S. Durfee, D. M. Kurn, and W. Ketterle, Phys. Rev. Lett. 75, 3969 (1995).

[3] C. C. Bradley, C. A. Sackett, J. J. Tolett, and R. G. Hulet, Phys. Rev. Lett. 75, 1687 (1995); C. C. Bradley, C. A. Sackett, and R. G. Hulet, Phys. Rev. Lett. 78, 985 (1997).

[4] N. N. Bogoliubov, J. Phys. (Moscow) 11, 23 (1947).

[5] E. P. Gross, Nuovo Cimento 20, 454 (1961); L. P. Pitaevskii, Sov. Phys. JETP 13, 451 (1961).

[6] D. A. W. Hutchinson, E. Zaremba, and A. Griffin, Phys. Rev. Lett. 78, 1842 (1997).

[7] F. Dalfovo, S. Giorgini, L. Pitaevskii, and S. Stringari, to appear in Rev. Mod. Phys. (1998).

[8] E. L. Pollock and D. M. Ceperley, Phys. Rev. B30, 2555 (1984); B36, 8343 (1987); D. M. Ceperley, Rev. Mod. Phys. 67, 1601 (1995).

[9] W. Krauth, Phys. Rev. Lett. 77, 3695 (1996).

[10] V. V. Goldman, I. F. Silvera, and A. J. Leggett, Phys. Rev. B 24, 2870 (1981).

[11] S. Giorgini, L. P. Pitaevskii, and S. Stringari, Phys. Rev. A 54, R4633 (1996).

[12] P. Grüter, D. Ceperley, and F. Laloë, Phys. Rev. Lett. 79, 3549 (1997).

[13] M. Bijlsma and H. T. C. Stoof, Phys. Rev. A 54, 5085 (1996).

[14] M. Houbiers, H. T. C. Stoof, and E. A. Cornell, Phys. Rev. A 56, 2041 (1997).

[15] R. P. Feynman, Statistical Mechanics (Benjamin/Cummings, Reading, MA, 1972).

[16] S. Giorgini, L. P. Pitaevskii, and S. Stringari, J. Low Temp. Phys. 109, 309 (1997); F. Dalfovo, S. Giorgini, M. Guilleumas, L. Pitaevskii, and S. Stringari, Phys. Rev. A 56, 3840 (1997).

[17] M. Holzmann and Y. Castin (unpublished); M. Naraschewski and R. J. Glauber (unpublished).

[18] The QMC data shown were obtained with $\tau=0.005 / \hbar \omega$. A minute systematic shift subsists.

[19] M. Holzmann and W. Krauth (unpublished).

[20] M.-O. Mewes, M. R. Andrews, N. J. van Druten, D. M. Kurn, D. S. Durfee, and W. Ketterle, Phys. Rev. Lett. 77, 416 (1996).

[21] J. R. Ensher, D. S. Jin, M. R. Matthews, C. E. Wieman, and E. A. Cornell, Phys. Rev. Lett. 77, 4984 (1996).

[22] The scaling behavior seems to be similar to an ideal gas, where finite-size effects lead to a deviation of the critical degeneracy parameter by $O\left(N^{-1 / 6}\right)$.

[23] W. H. Press S. A. Teukolsky, W. T. Vetterling, B. P. Flannery, Numerical Recipes, 2nd edition, Cambridge University Press (1992).

[24] The FORTRAN program for the fit procedure and the QMC code are made available (from $\mathrm{MH}$ or WK). 